\title{
Hyperintentionality Hypothesis of Major Depression. Disordered Emotional and Cognitive Self-Observation in Tripartite Synapses and the Glial Networks
}

\author{
Bernhard J Mitterauer* \\ Volitronics-Institute for Basic Research, University of Salzburg, Austria
}

${ }^{*}$ Corresponding authors: Bernhard J Mitterauer, MD, Emeritus Professor, Volitronics-Institute for Basic Research, University of Salzburg, Autobahnweg 7, A-5071 Wals, Austria, Tel: +4366-2851-039, Fax: +4366-28044-3861, E-mail: mitterauer.b@gmail.com

\begin{abstract}
Major depressive disorder is basically a disorder of the integration of distinct time periods generated in tripartite synapses and the astroglial networks. If the sequence of time periods is dysregulated, the patient does not only suffer from emotional impairment but also from cognitive deficits dependent on the brain region affected. Since the synaptic interactions operate on feedforward and feedback mechanisms, I speak of synaptic locations of self-observation in real time periods, information processing time periods and information integration time periods. If connexins in the astroglial network are overexpressed, the number of astrocytic receptors in tripartite synapses is also overexpressed. In the case of underexpression of connexins the expression of astrocytic receptors is decreased. In both states glial-neuronal interactions are dysregulated, incapable of processing and integrating environmental information in real time.

The hippocampus and the prefrontal cortex represent preferred brain regions of emotion generation and cognitive operations. In the hippocampal astroglial network both gap junctions and astrocytic receptors may be overexpressed and, as a consequence, transmitter substances cannot exert their balancing effects. Such a pathophysiological state operates hyperintentionally, since intentional programs generated in the astroglial network are not feasible in real time. Synaptic information processing is protracted and self-observation cannot take place in real time.

Inversely, in the prefrontal cortex gap junctions and astrocytic receptors are underexpressed causing shortened synaptic information processing and synaptic information flooding that cannot be completely integrated in intentional programming of the astroglial network. This causes retardation of thought processes. Emotional and cognitive impairment is experienced as depressed mood. Effects of antidepressant drugs on balancing glial-neuronal interactions may be basically caused by the expression of astroglial connexin proteins representing a promising target of treatment.
\end{abstract}

\section{Keywords}

Major depression, Pathophysiology, Glial-neuronal interaction, Hyperintentionality, Disordered self-observation, Treatment

\section{Introduction}

Major depression is a worldwide psychobiological disorder [1]. The basic symptoms are depressed mood and loss of interest or pleasure [2]. I will primarily focus on depressed mood, emotional and cognitive impairment. In the present paper the underlying pathophysiology and its interpretation is outlined.

The brain consists of a double cell structure, the neuronal cell system and the glial cell system. Various functions of glial cells have been identified with growing evidence that glia play an important role in their interaction with neurons. Here, I focus on glial-neuronal synaptic units, termed tripartite synapses, and the glial network. A tripartite synapse is composed of the neuronal component (preand postsynapse) and the astrocyte as the glial component. The interactions occur via transmitter substances, transporters, second messengers, ions, etc. Extracellular receptors also operate in glialneuronal interactions.

The glial network of astrocytes is organized in domains connected by gap junctions that embody mainly non-overlapping territories [3]. Gap junctions in the astrocytic network are built by connexin proteins composed of two connexons and conducting channels. Hemichannels (connexons) exert special conducting functions. In the astrocytic network connexin $43(\mathrm{Cx} 43)$ and connexin 30 (Cx30) represent the constituting proteins [4].

Intentionality is defined as follows: an intentional program generates a specific multirelational structure in the inner and outer environment based on the principle of that program [5]. Selfobservation measures environmental information commanded and controlled by experience-based intentional programs in preferred locations in the brain. Dysregulation of self-observation and hyperintentionality are elaborated in the hypothetical model proposed.

\section{Hypothetical model of major depression}

Major depression is basically a disorder of the integration of distinct time periods generated in tripartite synapses and the glial networks. If the sequence of time periods is dysregulated, the patient 
does not only suffer from emotional impairment, but also from deficits in cognitive function. Normally the activation of the astrocyte by the neuronal component of the synapse is processed in the millisecond to second, second to minute, and minute to hour or longer range of glialneuronal interactions. Since these synaptic interactions operate on feedforward and feedback mechanisms, I speak of synaptic locations of self-observation in real time periods, information processing time periods and information integration time periods. The glial network is composed of gap junctions interconnecting an astrocytic domain organization within a single astrocyte and between astrocytic domain organizations into non-overlapping territories.

I hypothesize that if the connexins in the astrocytic network are overexpressed, the number of astrocytic receptors is also overexpressed. In the case of underexpression of connexins, the expression of astrocytic receptors is decreased. In both states glialneuronal interactions are dysregulated, incapable of processing and integrating environmental information in real time.

The hippocampus and the prefrontal cortex represent preferred brain regions of emotion generation and cognitive operations. In the hippocampal astrocytic network gap junctions as well as astrocytic receptors in tripartite synapses may be overexpressed, and as a result neurotransmitter substances cannot exert their balancing effects. The overexpression of connexins in gap junctions generates intentional programs that are not feasible in real time. Such a pathophysiological state operates hyperintentionally. Since synaptic information processing is protracted, the patients' self-observation is not appropriate to his/her intentional programs.

Inversely, in the prefrontal cortex gap junction proteins and astrocytic receptors are underexpressed, causing a shortened synaptic information processing. Astrocytic receptors are stressed by neuronal activations incompletely processed in the astrocytic network due to reduced gap junction proteins (connexins, $\mathrm{Cx}$ ). This kind of information flooding cannot be integrated in the intentional programming of the gap junction network. As a consequence, thought processes are retarded, as for instance in monosyllabic speech. The impairment of emotion generation and cognitive function may be a result of incomplete self-observations with regard to intentional programs and environmental information, experienced as depressed mood.

\section{Outline of a tripartite synapse and the astroglial network}

Tripartite synapse: The basic anatomical components of a tripartite synapse are composed of the presynaptic neuron, the postsynaptic neuron, and the astrocyte embodying the glial cell with a synaptic cleft in between. The glial-neuronal interactions in chemical tripartite synapses occur via neurotransmitters, gliotransmitters and other substances (neuromodulators, neurotransporters, ions, etc.). Experimental neurophysiological research has demonstrated that the glial system exerts a modulatory function in its interactions with the neuronal system [6]. Since the topic of the present study is focused on tripartite synapses and the astrocytic network, other main glial structures and functions (oligodendroglia, myelin, microglia, etc.) are not considered. Figure 1 shows a schematic diagram of glial-neuronal interactions in the tripartite synapse [7].

Sensori-motor networks compute environmental information activating the presynapse [1]. The activated presynapse releases neurotransmitter substances (NT) occupying both postsynaptic receptors (por) and receptors on the astrocyte (acr) [2]. NT also activate gap junctions (g.j.) in the astrocytic network (syncytium) enhancing the spreading of calcium waves $\left(\mathrm{Ca}^{2+}\right)$ [3]. In parallel, the occupancy of acr by NT also activates $\mathrm{Ca}^{2+}$ within the astrocyte [4]. This mechanism exerts the production of gliotransmitters (GT) $[5,6]$ within the astrocyte. The occupancy of extrasynaptic and pre- and postsynaptic receptors (psr, por) by GT is excitatory [7] and on extrasynaptic por inhibitory [8]. Neurotransmission is also

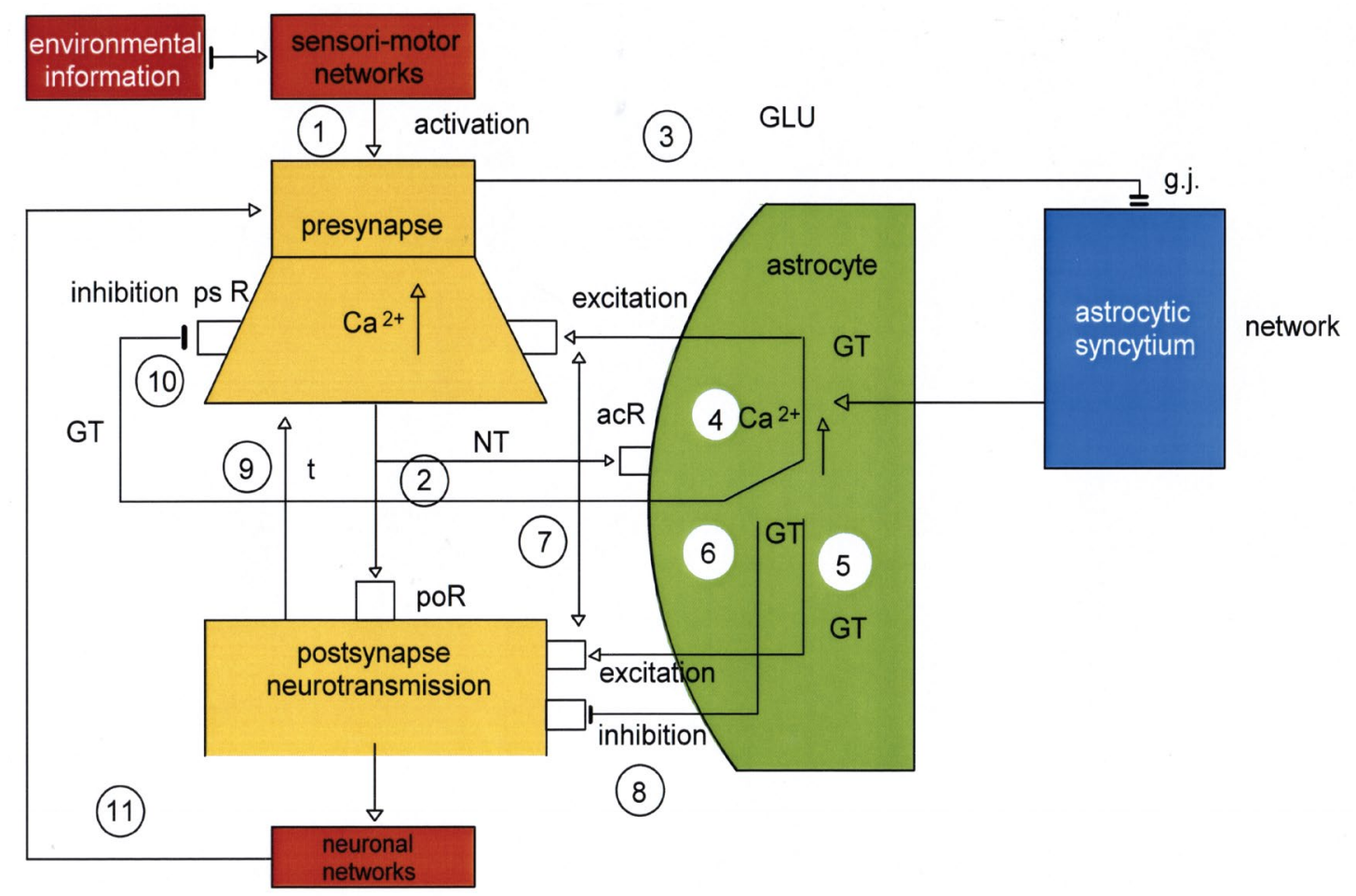

Figure 1: Schematic diagram of possible glial-neuronal interactions at the glutamatergic tripartite synapse.

Sensori-motor networks compute environmental information activating the presynapse [1]. The activated presynapse releases glutamate (GLU) occupying both postsynaptic receptors (poR) and receptors on the astrocyte (acR) [2]. GLU also activates gap junctions (g.j.) in the astrocytic syncytium, enhancing the spreading of $\mathrm{Ca}^{2+}$ waves [3]. In parallel, the occupancy of acR by GLU also activates $\mathrm{Ca}^{2+}$ within the astrocyte [4]. This mechanism exerts the production of $\mathrm{GLU}$ [5] and adenosinetriphosphate (ATP) [6] within the astrocyte, now functioning as gliotransmitters, whereas the occupancy of the extrasynaptic and pre- and postsynaptic receptors by GLU is excitatory [7], the occupancy of these receptors by ATP is inhibitory [8]. In addition, neurotransmission is also inactivated by the reuptake of GLU in the membrane of the presynapse mediated by transporter molecules (t) [9]. ATP inhibits the presynaptic terminal via occupancy of cognate receptors (prR) temporarily turning off synaptic neurotransmission in the sense of a negative feedback [10]. Synaptic information processing is transmitted to neuronal networks activating the synapse again [11]. 
inactivated by the reuptake of NT in the presynaptic membrane mediated by transporter molecules ( $\mathrm{t}$ ) [9]. Moreover, GT inhibit the presynaptic terminal via occupancy of psr temporarily turning off synaptic transmission in the sense of a negative feedback [10]. Synaptic information processing can be transmitted to the neuronal network activating the synapse again [11].

Astroglial network: The biological structure proposed focuses on gap junctions between astrocytes, the main glial cell type beside oligodendrocytes and microglia. Gap junctions provide a structural link by which single cells are coupled to build a functional network, called syncytium, with communication dynamics that cannot be exerted by individual cells. Gap junctions of an astrocytic syncytium consist of connexins forming gap junction channels by hemichannels of different kinds [4]. Whereas astrocytes are interconnected with their neighbors via gap junctions, the interactions of astrocytes with neurons occur mainly in tripartite synapses [8].

The number and composition of gap junctions can be dynamically regulated by upregulating connexin biosynthesis or decreasing the rate of connexin degradation in the endoplasmic reticulum, and by enhancing gap junction assembly. If gap junctions are frequently coupled within time scales of seconds or hours, they form plaques [4].

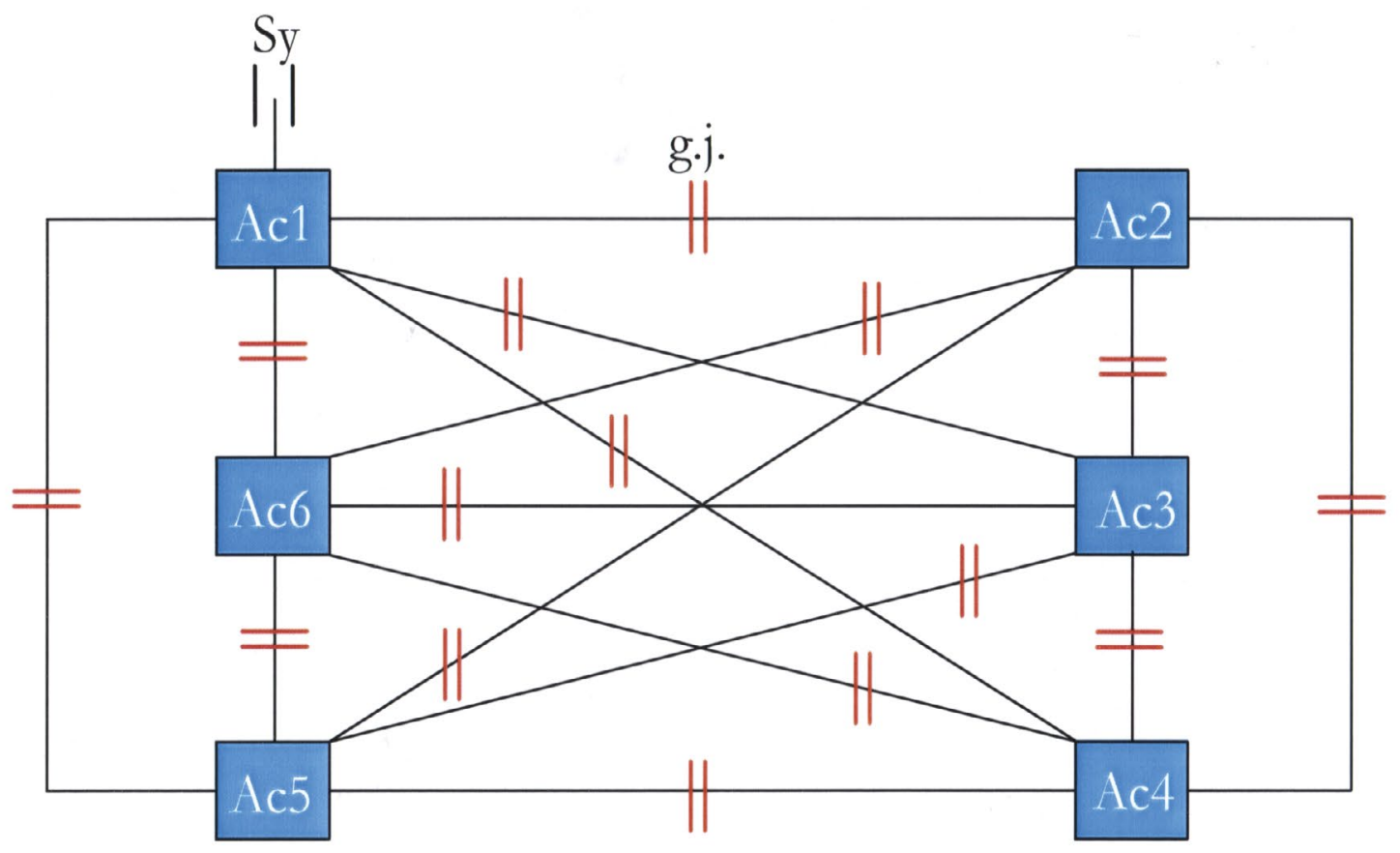

Figure 2: Outline of an astrocytic syncytium.

Six astrocytes $\left(\mathrm{Ac}_{1} \ldots \mathrm{Ac}_{6}\right)$ are interconnected via fifteen gap junctions (g.j.) building a complete syncytium. Each astrocyte contacts a neuronal synapse (tripartite synapse). For the sake of clarity, only one synaptic contact is shown (Sy).

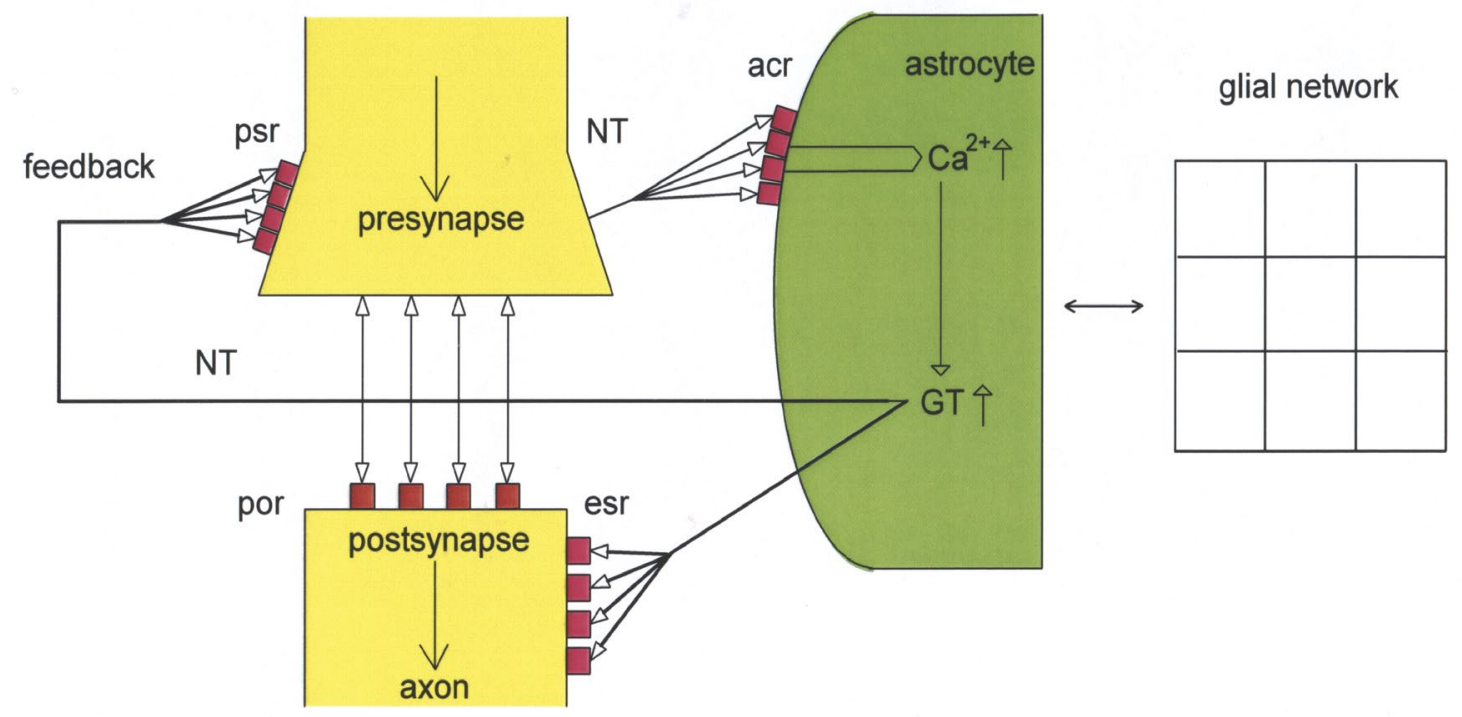

Figure 3: Schematic diagram of a balanced tripartite synapse and the glial network.

Neurotransmitters (NT) occupy astrocytic receptors (acr) and postsynaptic receptors (por) released from the presynapse (double headed arrows: activation and reuptake). The activation of acr by NT activates $\mathrm{Ca}^{2+}$ that interacts with the glial network (depicted as lattice). Activated Ca ${ }^{2+}$ causes the production of gliotransmitters (GT) that occupy extrasynaptic receptors (esr) and in parallel exert a feedback to presynaptic receptors (psr). The balance of receptors and transmitter substances enables a normal synaptic information processing. 
Figure 2 shows a diagrammatic schema depicting an astrocytic syncytium (a). Six astrocytes $\left(A_{c 1} \ldots A_{c 6}\right)$ are completely interconnected via fifteen gap junctions (g.j.). Each astrocyte contacts a neuronal synapse building a tripartite synapse ( $\mathrm{tSy}$, only one is shown). This simple diagram refers to the elementary components and their connections in the astrocytic network [9]. Based on this diagram of the astroglial network I have described a formalism of intentional programming in these networks [5]. Generation of pathways via gap junctions is interpreted by intentional programming which may command and control information processing of tripartite synapses. Balanced and imbalanced glial-neuronal interactions in
tripartite synapses and the astrocytic network

Balanced glial-neuronal interaction: Figure 3 represents a schematic diagram in which pre- and postsynaptic receptors and astrocytic receptors are balanced via neuronal and glial transmitter substances. The gap junction network depicts a lattice (gliotransmitters) that represents a structure appropriate to synaptic information processing. The basic mechanisms operate balanced in the tripartite synapse as shown in figure 1.

\section{Imbalanced information processing:}

Hippocampus: The hippocampus embodies a mainly emotion generating brain region. Figure 4 depicts an overexpressed gap junction network (increased interconnecting lines compared to figure 3). Astrocytic receptors are overexpressed. Overexpressed astrocytic receptors cause an incomplete activation by neuronal transmitter substances (dashed lines). This relative lack of neurotransmitter substances (NT) and the decreased production of gliotransmitters (GT) protract the feedback mechanism on cognate presynaptic and extracellular receptors (dashed lines). Such a disorder implies the interpretation that the gap junction network and the astrocytic receptors function hyperintentionally. Given a tripartite synapse as a self-reflexive system [10], self-observation does not operate in real time but is dysregulated. Together, these pathophysiological mechanisms may be fundamentally responsible for emotional impairment in depression.

Prefrontal cortex: The prefrontal cortex is specialized for cognitive operations. Figure 5 outlines an underexpressed gap junction network (decreased interconnecting lines) and underexpressed astrocytic receptors. Contrarily to the hippocampal imbalance the production of neurotransmitters (NT) overloads astrocytic receptors. The same flooding occurs by gliotransmitters (GT) on presynaptic and extrasynaptic receptors, because of their increased production generated by overactivated $\mathrm{Ca}^{2+}$ waves through NT. GT flooding of cognate presynaptic and extrasynaptic receptors shorten the feedback function of synaptic information transmission. In addition, these pathophysiological operations are based on incomplete intentional programming in the gap junction network and on shortened self-observation incomplete for integrative synaptic information processing. Together, the imbalances in the prefrontal cortex described may be responsible at least in part for cognitive impairment in major depression.

\section{Effects of antidepressant drugs}

Growing experimental evidence indicates effects of antidepressant drugs on both neuronal and astrocytic transporters inhibiting transmitter re-uptake. Generally, various impairments of the glial cell system in major depressive disorder have been identified $[11,12]$. Experimental research mainly investigates mechanisms of selective serotonin re-uptake inhibitors (SSRI) in neuronal synapses and their networks in various brain regions [13]. The search for common mechanisms working in synaptic imbalances and network dysregulations that may be responsible for major depressive disorder and the pathophysiology of neurological and neuropsychiatric brain disorders as a whole represents a severe problem.

A mostly recent study based on excellent methodology may support my model of the pathophysiology of major depression. Quesseveur and coworkers [14] investigated the role of hippocampal astroglial connexin 43 in emotionality and the effects of SSRIs and stress. The main result is this: considering that phosphorylation is a prerequisite for acute function of connexins [15], the therapeutic effects of antidepressant drugs might implicate the functional inactivation of $\mathrm{Cx} 43$. However, other data show that chronic treatment with antidepressant drugs such as fluoxetine or duloxetine increases Cx43 gap junction coupling [16]. In addition, functional imaging studies of patients with depression indicate that the clinical response of fluoxetine is a decreased functional activity of limbic regions including the hippocampus and, on the other hand, an increased functional activity in the cortex [17]. Hence, it is decisive which brain region is referred to concerning the mechanism of phosphorylation, i.e. hippocampus or prefrontal cortex. Hippocampal and cortical

\section{Hippocampus}

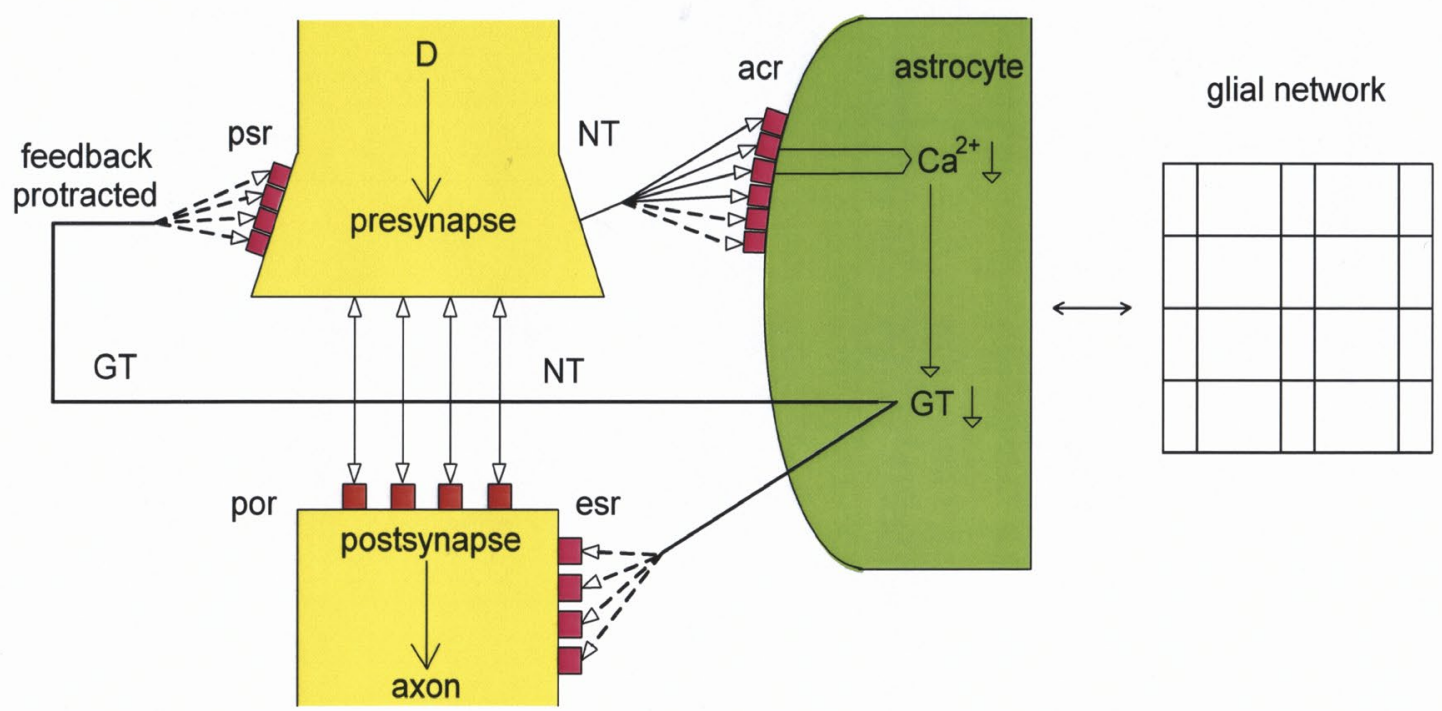

Figure 4: Outline of an overexpressed glial network and overexpressed astrocytic receptors.

Gap junctions in the glial network are overexpressed (increase of interconnecting lines) as well as astrocytic receptors (acr). A relative lack of neurotransmitters (NT) cannot occupy acr (dashed lines) so that Ca2+ activation and gliotransmitter (GT) production is decreased. This leads to a protracted feedback on presynaptic receptors (psr) (dashed lines). The activation of extrasynaptic receptors (esr) is also protracted (dashed lines). 


\section{Prefrontal cortex}

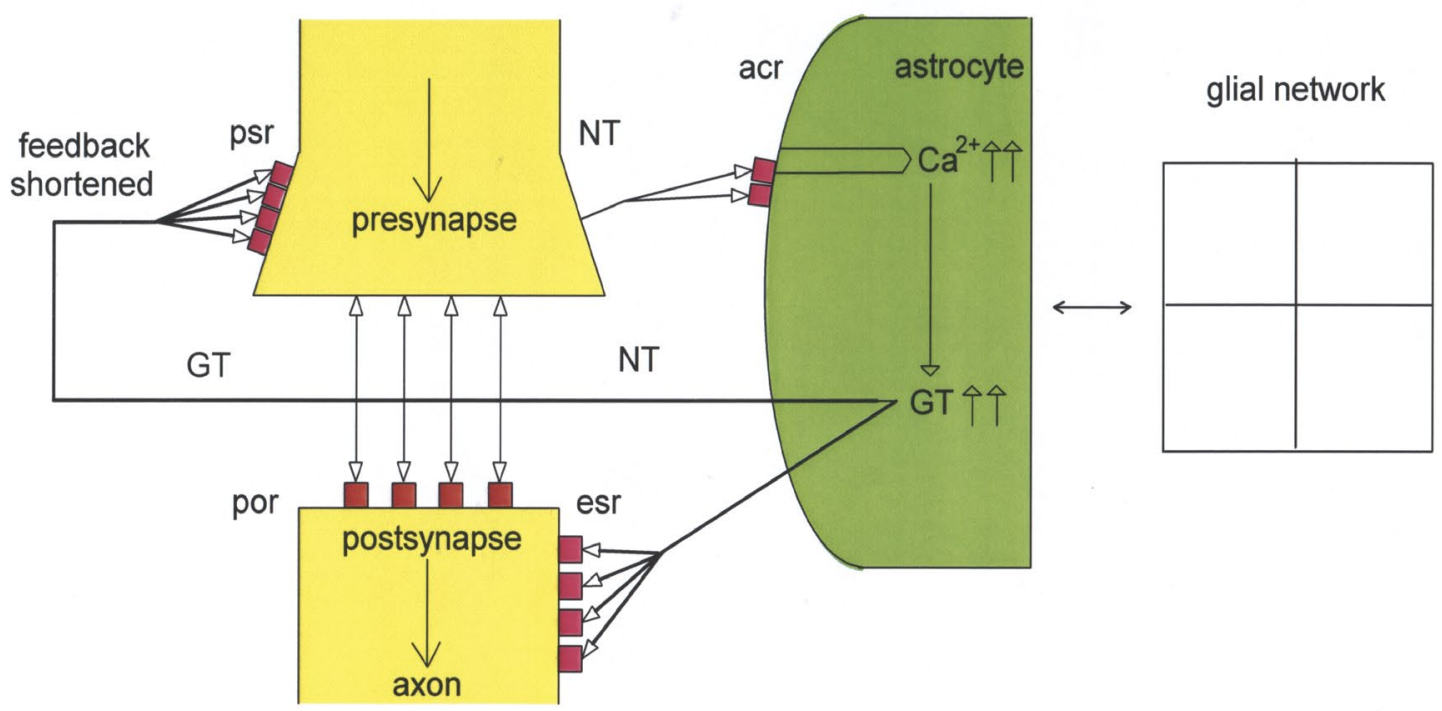

Figure 5: Outline of an underexpressed glial network and underexpressed astrocytic receptors.

Gap junctions in the glial network are underexpressed (decrease of interconnecting lines) as well as astrocytic receptors (acr). Neurotransmitters (NT) overactivate (increase of lines) acr and calcium ions (Ca2+) causing an increased production of gliotransmitters (GT). This leads to a shortened feedback on presynaptic receptors (psr) and overactivation of extrasynaptic receptors (esr).

astrocytes may have different impact on neuronal activity dependent on their cellular and molecular environment. This implies that therapeutic responses similar to antidepressant-like effects are based on activation of $\mathrm{Cx} 43$ gap junction channels in the cortex but on inactivation in the hippocampus. Moreover, the expression of $\mathrm{Cx} 43$ [18] suggests that enhanced hemichannel activity in astrocytes might contribute to dysfunction in emotional brain circuits [14].

The most important result of this study discussed is that the degree of $\mathrm{Cx} 43$ phosphorylation influences the expression of connexin proteins with different effects on overexpressed and underexpressed astrocytic gap junction networks. In this case my pathophysiological model of depression may explain why the fundamental therapeutic activity of antidepressant drugs on $\mathrm{Cx} 43$ hemichannel activity in emotion generating regions of the hippocampus exerts a decreased expression, whereas the activity of $\mathrm{Cx} 43$ resulting from a lower level of phosphorylation exerts an increased production of $\mathrm{Cx} 43$ proteins in the cognition processing region of the prefrontal cortex. These mechanisms may be elementary for balancing imbalances in tripartite synapses and their networks (Figure 3 and Figure 5).

In addition, there is evidence that SSRIs inhibit 5-HT uptake through blockade of neuronal and glial serotonin transporters with the subsequent increase of extracellular 5-HT levels contributing to activate astroglial 5-HT receptors [19], which in turn might directly regulate $\mathrm{Cx}$ function or expression. Since these balancing mechanisms in synaptic glial-neuronal interactions may be based on different phosphorylation degrees in emotion and cognition processing regions in brains with depression, new substances that target phosphorylation in pertinent brain regions may be very promising. In this context, Autry and coworkers [20] found that ketamine-mediated $\mathrm{N}$-methyl-D-asparate receptor (NMDAR) blockade at rest deactivates eukariotic elongation factor 2 (eEF2) kinase resulting in reduced eEF2 phosphorylation and depression of brain-derived neurotrophic factor (BDNF) translation inducing fast-acting behavioral antidepressant-like effects. It is concluded that protein synthesis regulation (phosphorylation) by spontaneous neurotransmission may serve as a viable therapeutic target for fast-acting antidepressant development. However, this study does not explicitly refer to neuronal-glial interactions and it lacks a comprehensive pathophysiological model.

\section{Intentionality and self-observation dysregulated in major depression}

Mechanisms guiding thought and action in accordance with internal intentions are described especially in neuroscience of will action [21]. In the pathophysiology of psychobiological disorders actions driven by a current environmental stimulus may be incompatible with an individual's goals, yet the regulation or control of these distinct action pathways is necessary in order to act in a goal-directed manner [22]. In normal glial-neuronal interactions the glial network may generate intentional programs [5] that select appropriate information from the neuronal component determined by experiences in distinct time scales [10]. In biological language of experimental research [23] tripartite synapses embody measurement instruments of environmental information commanded and controlled by the internal state of the glial network at interaction time $\mathrm{x}$. In addition, the organization within a single astrocyte operates as a reflexive system that offers to characterize tripartite synapses as startpoints of self-observation [24]. Since the pattern of an intentional program determines the feasibility of complete self-observation, over-or underexpressed glial gap junction networks cause disorders of self-observation.

In depression the overexpressed glial network and astrocytic receptors operate hyperintentionally in emotion processing regions. Therefore, the patients are incapable of integrating actual information from the environment in real time. Since this physiological dysregulation basically occurs in the hippocampus and related regions, a patient with depression is not emotionally affected by actual informations or events in the environment, in the sense of emotional retardation. In parallel, cognition processing areas as the prefrontal cortex may be impaired by underexpressed gap junction networks and astrocytic receptors. Here, environmental informations cannot be "grasped" in momentum, so that the patient is irritated by information flooding and selfobservation is fragmented, leading to agitated self-observation and behavior. Together, in major depressive disorder emotional dysregulation may be caused by protracted self-observation and cognitive dysregulation by shortened self-observation. Both kinds of information processing are incomplete and result in emotional retardation, cognitive agitation, and in various known symptoms on the behavioral level. 


\section{Testing the theory and final remarks}

First of all, testing the theory proposed requests a questionnaire for exploring the intentional state of a patient suffering from depression. In the case of hyperintentionality non-feasibility of intentions may basically cause a depressive mood. Positron Emission Tomography (PET) is a promising approach for in vivo quantification of over- or underexpressed gap junctions responsible for imbalanced information processing in glial networks of the hippocampus and prefrontal cortex. Currently, it is possible to quantify synaptic density in the living brain using synaptic glycoprotein $2 \mathrm{~A}$ radioligand $((11) \mathrm{C})$ UBC-J combined with PET [25]. Although Finnema and coworkers [26] use synaptic vesicle glycoprotein $2 \mathrm{~A}$, it should be possible to apply connexin protein 43 for testing my model of major depressive disorder.

My approach to depression research is based on interdisciplinary science. The pathophysiological model dates back to 2004 [27] with subsequent elaborations according to current experimental results. The concept of intentionality plays an important role in neuroscience whereas the concept of self-observation is neglected, at least with concern to glial-neuronal interactions. However, in all areas of interdisciplinary research an understanding of brain structures and functions requires a fundamental theory of self-observation. The proposed hypothetical model might be explanatory with regard to the pathogenesis of basic symptoms of major depression [28] Most importantly, improvements of biological treatment should be possible. Halassa and Haydon [29] suggested that first-treatment of a major brain dysfunction will be through small molecules on biologically targeted glia. I suppose that phosphorylation may provide a promising step in this direction.

\section{Acknowledgement}

I am very grateful to Birgitta Kofler-Westergren for preparing the final version of the paper.

\section{References}

1. Milanovic SM, Erjavec K, Poljicanin T, Vrabec B, Brecic P (2015) Prevalence of depression symptoms and associated socio-demographic factors in primary health care patients. Psychiatr Danub 27: 31-37.

2. American Psychiatric Association (2013) Diagnostic and statistical manual of mental disorders. Washington DC, USA.

3. Oberheim NA, Takano T, Han X, He W, Lin JH, et al. (2009) Uniquely hominid features of adult human astrocytes. J Neurosci 29: 3276-3287.

4. Ransom BR, Giaume C (2013) Gap junctions, hemichannels. In: Kettenmann H, Ransom BR, Neuroglia, Oxford University Press, New York, USA, 292305 .

5. Mitterauer B (2007) Where and how could intentional programs be generated in the brain? A hypothetical model based on glial-neuronal interactions. Biosystems 88: 101-112.

6. Haydon PG, Carmignoto G (2006) Astrocyte control of synaptic transmission and neurovascular coupling. Physiol Rev 86: 1009-1031.

7. Mitterauer BJ (2012) Qualitative information processing in tripartite synapses. Cogn Comp 4: 181-194.

8. Araque A, Parpura V, Sanzgiri RP, Haydon PG (1999) Tripartite synapses: glia, the unacknowledged partner. Trends Neurosci 22: 208-215.
9. Mitterauer BJ (2010) Syncytiopathy hypothesis of depression: downregulation of glial connexins may protract synaptic information processing and cause memory impairment. Med Hypotheses 74: 497-502.

10. Mitterauer BJ (2015) Self-structuring of motile astrocytic processes within the network of a single astrocyte. Adv Biosci Biotech 6: 723-733.

11. Czéh B, Di Benedetto B (2013) Antidepressants act directly on astrocytes: evidences and functional consequences. Eur Neuropsychopharmacol 23 : 171-185.

12. Verkhratsky A, Rodríguez JJ, Steardo L (2014) Astrogliopathology: a central element of neuropsychiatric diseases? Neuroscientist 20: 576-588

13. Caplan JD, Gopinat S, Abdallah CG, Berry BR (2014) A neurobiological hypothesis of treatment-resistant depression-mechanisms for selective serotonin reuptake inhibitors non-efficacy. Front Behav Neurosci 8: 189.

14. Quesseveur G, Portal B, Basile JA, Ezan P, Mathou A, et al. (2015) Attenuated Levels of Hippocampal Connexin 43 and its Phosphorylation Correlate with Antidepressant- and Anxiolytic-Like Activities in Mice. Front Cell Neurosci 9: 490.

15. Solan JL, Lampe PD (2009) Connexin43 phosphorylation: structural changes and biological effects. Biochem J 419: 261-272.

16. Sun JD, Liu Y, Yuan YH, Li J, Chen NH (2012) Gap junction dysfunction in the prefrontal cortex induces depressive-like behaviors in rats. Neuropsychopharmacology 37: 1305-1320.

17. Mayberg HS, Brannan SK, Tekell JL, Silva JA, Mahurin RK, et al. (2000) Regional metabolic effects of fluoxitine in major depression: serial changes and relationship to clinical response. Biol Psych 48: 830-843.

18. Hausbein R, Trotter J, Behl C, Clement AB (2009) Increased connexin 43 expression as a potential mediator of the neuroprotective activity of the corticotropin-releasing hormone. Med Endocrinol 23: 1479-1493.

19. Quesseveur G, David DJ, Gaillard MC, Pla P, Wu MV, et al. (2013) BDNF overexpression in mouse hippocampal astrocytes promotes local neurogenesis and elicits anxiolytic-like activities. Transl Psych 3: e253.

20. Autry AE, Adachi M, Nosyreva E, Na ES, Maarten F Los, et al. (2011) NMDA receptor blockade at rest triggers rapid behavioural antidepressant response. Nature 474: 91-95

21. Cohen JD, Botvinick M, Carter CS (2000) Anterior cingulate and prefrontal cortex: who's in control? Nat Neurosci 3: 421-423.

22. Fabre D, Vehier A, Chesnoy-Servanin G, Gouiller N, Thierry D'Amato, et al. (2015) From theory to prACTice: a cognitive remediation program based on a neuropsychological model of schizophrenia. Front Psych 6: 169.

23. Kimelberg HK (2012) Mature protoplasmic mammalian astrocytes Morphology, interrelationships and implications for function. In: Scemes E, Spray DC, Astrocytes wiring the brain. CRC Press, Front Neurosci. Boca Raton, USA, 3-24.

24. Giaume C (2012) Neuroglial networks: glial wiring also matters. In: Scemes E, Spray DC, Astrocytes wiring the brain. Front Neurosci, Boca Raton, USA, 139-156.

25. Finnema SJ, Nabulsi NB, Eid T, Detyniecki K, Lin SF, et al. (2016) Imaging synaptic density in the living human brain. Sci Transl Med 8: 348ra96.

26. Mitterauer B (2004) Imbalance of glial-neuronal interaction in synapses: a possible mechanism of the pathophysiology of bipolar disorder. Neuroscientist 10: 199-206.

27. Baer W (2015) On the necessity of including the observer in physical theory. Cosmos and History: The Journal of Natural and Social Philosophy 11: 160-174.

28. Mitterauer BJ (2009) Narziss und Echo. Ein psychobiologisches Modell der Depression. Springer, Vienna, New York, USA.

29. Halassa MM, Haydon PG (2013) Astrocyte modulation of mammalian synapses:circuits and behaviors. In: Kettenmann H, Ransom BR, Neuroglia, Oxford University Press, New York, USA, 494-503. 